\title{
Generator output and static capacitor control considering voltage stability for large penetration of photovoltaic power
}

\author{
Atsushi Enomoto ${ }^{\mathrm{a}}$, Shunsuke Aida ${ }^{\mathrm{a}^{*}}$, Shinichi Iwamoto ${ }^{\mathrm{a}}$ \\ Satoshi Osaki ${ }^{\mathrm{b}}$, Nobuyuki Ariyoshi ${ }^{\mathrm{b}}$, Kimihiko Shimomura $^{\mathrm{b}}$ \\ ${ }^{a}$ WASEDA University, 3-4-1 Okubo Shinjuku-ku, Tokyo 169-8555, Japan \\ ${ }^{b}$ CHUBU Electric Power Company, Japan
}

\begin{abstract}
In Japan, the introduction of large capacity of clean energy such as PV (photovoltaic power generation) is planned to reduce environmental burdens. The Japanese government has set the target of $53 \mathrm{GW}$ of PV by 2030 . However, large penetration of PV will cause several problems in power systems. One of these problems is that voltage values increase with the amount of PV penetration. Thus, we focus our attention on the upper voltage limit for a large penetration of $\mathrm{PV}$ in terms of voltage stability. In this paper, we consider a smart generator output and static capacitor control for the large penetration of PV. For the generator output control we propose to use the optimal power flow in terms of minimizing bus voltage deviations from the prescribed values. Simulations are run using the IEEJ WEST 10machine $\mathrm{O} / \mathrm{V}$ system model to confirm the validity of the proposed method.
\end{abstract}

Keywords: Voltage stability, VMPI, generator output control, static capacitor control, photovoltaic power

\section{Introduction}

Since power system loads are located further away from power plants and have become more unevenly distributed in recent years, it has also become more difficult to maintain voltage stability in power systems. Therefore, proper voltage ranges are determined in order to prevent voltage instability and to protect power devices. In our previous studies of a voltage stability control scheme, we proposed a voltage stability index, the voltage margin proximity index (VMPI), that could easily yield the voltage stability margin [1], [2]. In the later stages of the study, to prevent voltage instability phenomena caused by contingency or voltage collapse caused by heavy loads, we proposed a static voltage stability preventive control scheme and an emergency voltage stability control scheme both based on VMPI [3][5].

Recently, however, clean energy such as photovoltaic power generation (PV) or wind power generation, is penetrating into the Japanese power system following the enforcement of the Renewable Portfolio Standard (RPS) law and the feed-in tariff (FIT) policy. If a large number of PV penetrates into the power system, voltage values increase, since load demand is decreased due to the active power outputs of the PV. Especially, for decreasing power demand in the spring (Japanese Golden Week) and summer (Japanese Religious Holidays), it is considered that the voltage rise will be remarkable. In this paper, we therefore focus our attention on the upper voltage limits for a large penetration of PV. We propose a smart generator output and static capacitor control for the large penetration of PV. For the generator output control we propose to use the optimal power flow in terms of minimizing bus voltage deviations from the prescribed values. In addition, if voltage value violates the voltage upper limit, we consider static capacitors as control equipment against high voltages, and propose a static capacitor

\footnotetext{
* Manuscript received May 15, 2013; revised July 29, 2013.

Corresponding author. Tel.: +81 3-5286-3184; E-mail address: iwamoto.lab.pwrs@gmail.com.
} 
control method by using VMPI. Simulations are run using IEEJ (the Institute of Electrical Engineers of Japan) WEST 10-machine O/V system model [6] to confirm the validity of the proposed method.

In this paper, we assume that generator output control means control of only active power from generators, and that the power factor of the PV is constant at 1.0. In addition, we focus our attention on a static analysis of voltage stability, and thus dynamics are not considered here.

\section{Voltage Stability Index}

\subsection{Voltage stability index VMPI}

VMPI is an index that evaluates the voltage margin from the operating point to the boundaries of the proper voltage range, especially the upper voltage limit. The concept behind VMPI is shown in Fig.1. VMPI is calculated based on the angle between vectors at the upper voltage limit $\left(y_{U}\right)$ and at the operating point $\left(y_{s}\right)$. VMPI is classified into two types; one based on the voltage space and the other on the specified space. In this paper, we use the latter, which is superior to the former in terms of its linearity.

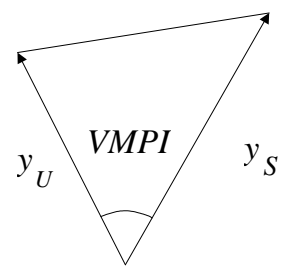

Fig. 1. Concept behind voltage stability index VMPI.

The vector at the operating point is specified by the initial value:

$$
\mathbf{y}_{s}=\left[\begin{array}{lllll}
e & f & P_{G e n} & V_{G e n}{ }^{2} P_{\text {Load }} & Q_{\text {Load }}
\end{array}\right]^{T} \text {. }
$$

The specified value at the upper voltage limit is obtained by an optimal power flow (OPF) calculation using the following formulae.

Objective function:

$$
\text { Minimize } f=k_{c}
$$

Equality constraints:

$$
\begin{array}{ll}
\text { Generator buses } & P_{G e n}=k_{c} \cdot P_{G e n}, V_{G e n}=V_{G e n}, \\
\text { Load buses } & P_{\text {Load }}=k_{c} \cdot P_{\text {Load }}, Q_{\text {Load }}=k_{c} \cdot Q_{\text {Load }} \\
\text { Slack bus } & e=e, f=f
\end{array}
$$

Inequality constraints:

Voltage limits

$$
\begin{array}{ll}
\text { Voltage limits } & V_{\text {min }}<V_{\text {Load }}<V_{\max } \\
\text { Reactive power limits } & Q_{\text {Gen min }}<Q_{\text {Gen }}<Q_{\text {Gen max }}
\end{array}
$$

where

$$
\begin{aligned}
e: & \text { real part of voltage } \\
f: & \text { imaginary part of voltage } \\
P_{G e n}: & \text { active power of generator } \\
V_{\text {Gen }}: & \text { voltage of generator } \\
P_{\text {Load }}: & \text { active power of load } \\
Q_{\text {Load }}: & \text { reactive power of load }
\end{aligned}
$$

$$
\begin{aligned}
k_{c}: & \text { load multiplier } \\
V_{\min }: & \text { lower voltage limit } \\
V_{\max }: & \text { upper voltage limit } \\
Q_{i}: & \text { reactive power at generator i } \\
Q_{\text {Gen_min }}: & \text { lower reactive power limit of generator } \\
Q_{\text {Gen_max }}: & \text { upper reactive power limit of generator }
\end{aligned}
$$

The specified vector at the operating point is $\left\|y_{U}\right\|>\left\|y_{s}\right\|$ when it is within the proper voltage range, 
and is $\left\|y_{U}\right\|<\left\|y_{s}\right\|$ when it falls below the upper voltage limit. Using these properties, VMPI is defined as follows:

$$
V M P I= \begin{cases}\cos ^{-1} \frac{y_{s}^{t} y_{U}}{\left\|y_{s}\right\| \cdot\left\|y_{U}\right\|}, & \left\|y_{U}\right\|>\left\|y_{s}\right\| \\ -\cos ^{-1} \frac{y_{s}^{t} y_{U}}{\left\|y_{s}\right\| \cdot\left\|y_{U}\right\|}, & \left\|y_{U}\right\|<\left\|y_{s}\right\|\end{cases}
$$

\subsection{VMPI sensitivity}

VMPI sensitivity shows the variation obtained by switching one of the voltage control device units. VMPI sensitivity VMPIs of a voltage control device is represented by

$$
V M P I s=V M P I^{\prime}-V M P I^{0}
$$

where $V M P I^{0}$ is the value of VMPI before the switch and $V M P I^{\prime}$ is the value of VMPI after the switch.

Fig. 2 shows VMPI variation, that is, VMPI sensitivity when the static capacitors (buses 6, 7 and 16) are controlled for the IEEJ WEST 10-machine O/V system model. In Fig. 3, it is established that the behaviour of VMPI is linearly dependent on the amount of control of the static capacitor. A similar linearity is also found when the other buses are controlled. Because there is linearity between VMPI and the control variable of the control devices, we can calculate the required control variables by using VMPI sensitivity.

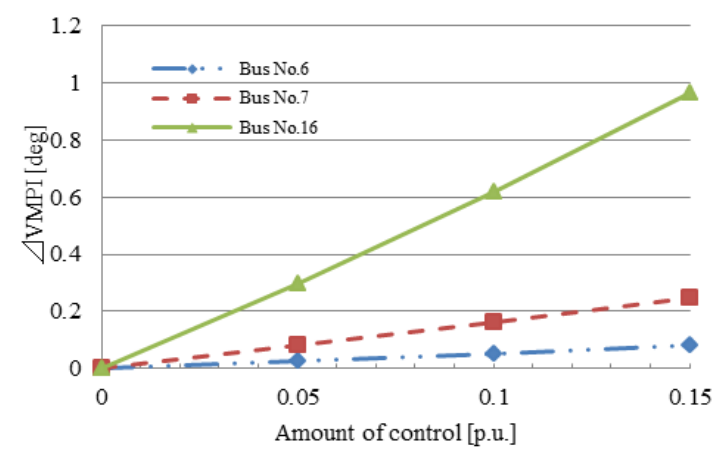

Fig. 2. VMPI sensitivity.

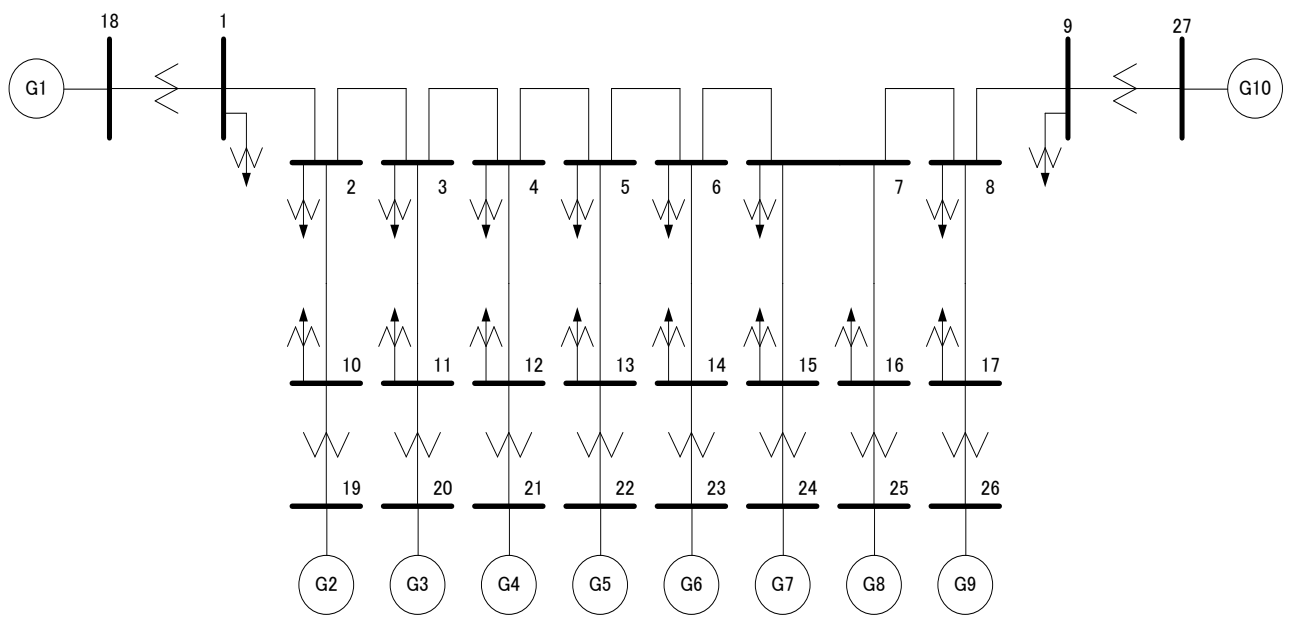

Fig. 3. IEEJ WEST 10- machine O/V system model. 


\section{Relationship between VMPI and Penetration of PV}

We demonstrate the relationship between VMPI and the penetration of PV in order to show the influence on the voltage stability when a large number of PV penetrate the power system model. We use the IEEJ WEST 10-machine O/V system model shown in Fig. 3 to run simulations.

The assumptions of the simulations are as follows:

1) PV is installed to all load buses, and the power output is proportional to each load demand.

2) Reductions in the generator active power outputs are the same as the total power of the PV.

3) The active power outputs are controlled for all generators, and reductions are proportional to each generator's original active power output.

In Fig. 4, when the installed PV a re at about 13 [p.u.], VMPI is below zero, and it is confirmed that the voltage value deviates from the proper voltage range of 1.05 [p.u.].

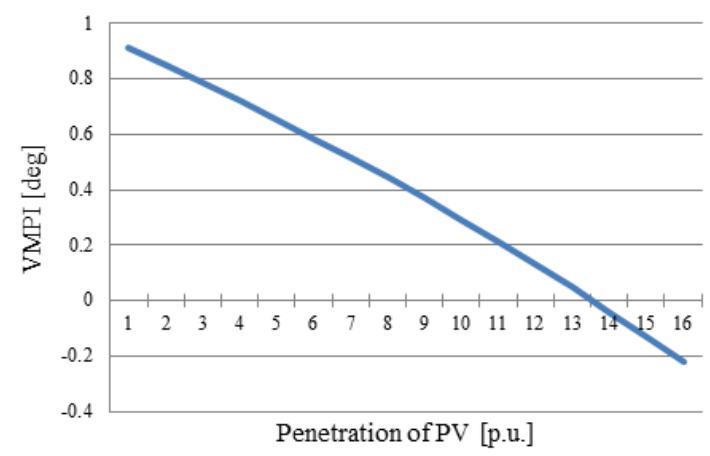

Fig. 4. Variation in VMPI for increasing penetration of PV.

\section{Generator Output Control Method Regarding Each Load Bus Voltage (Proposed Method)}

As stated previously, if we do not control the static capacitors with respect to the voltage, then the voltage values deviate from the proper voltage range when the level of installed PV reaches about 13 [p.u.]. Therefore, if a large number of PVs are to be installed in the power system, it is possible that the voltage values will deviate from the proper voltage range. Thus, in this section, a generator output control method is stated which uses VMPI for a large penetration of PV.

In the previous section, the active power output reductions are proportional to each generator's original active power output. However, this control method is not effective generator output control in terms of the voltage stability. Therefore, for the generator output control we propose to use the optimal power flow in terms of minimizing bus voltage deviations from the prescribed values.

Formulation of minimizing bus voltage deviations OPF

Objective function:

$$
\text { Minimize function }=\Sigma\left|V_{\text {ref }}-V_{\text {Load }}\right|
$$

Equality constraints:

$$
\begin{array}{ll}
\text { Generator buses } & V_{\text {Gen }}=V_{\text {Gen }} \\
\text { Load buses } & P_{\text {Load }}=P_{\text {Load }}, Q_{\text {Load }}=Q_{\text {Load }} \\
\text { Slack bus } & e_{\text {Slack }}=e_{\text {Slack }}, f_{\text {Slack }}=f_{\text {Slack }}
\end{array}
$$

Inequality constraints:

Voltage limits

Active power limits

Reactive power limits

$$
\begin{aligned}
& V_{\text {min }}<V_{\text {Load }}<V_{\text {max }} \\
& P_{\text {Gen min }}<P_{\text {Gen }}<P_{\text {Gen max }} \\
& Q_{\text {Gen min }}<Q_{\text {Gen }}<Q_{\text {Gen max }}
\end{aligned}
$$


where

$$
\begin{aligned}
V_{\text {ref }}: & \text { reference voltage } \\
V_{\text {Load }}: & \text { voltage at loads } \\
e: & \text { real part of voltage } \\
f: & \text { imaginary part of voltage } \\
P_{\text {Gen }}: & \text { active power of generator } \\
V_{\mathrm{Gen}}: & \text { voltage of generator } \\
P_{\text {Load }}: & \text { active power of load } \\
Q_{\text {Load }}: & \text { reactive power of load }
\end{aligned}
$$

$$
\begin{aligned}
V_{\min }: & \text { lower voltage limit } \\
V_{\max }: & \text { upper voltage limit } \\
P_{\text {Gen }}: & \text { active power of generator } \\
Q_{\text {Gen }}: & \text { reactive power of generator } \\
P_{\text {Gen_min }}: & \text { lower active power limit of generator } \\
P_{G e n \_m a x}: & \text { upper active power limit of generator } \\
Q_{\text {Gen_min }}: & \text { lower reactive power limit of generator } \\
Q_{\text {Gen_max }}: & \text { upper reactive power limit of generator }
\end{aligned}
$$

Also, it is considered that the voltage value violates the voltage upper limit only using the generator output control. Therefore, we control the static capacitor if the voltage value deviates from the voltage upper limit. In this paper, the flowchart of PV penetration is shown in Fig. 5. At first, we install 1 [p.u.] PV and determine the generator output by calculating the OPF. Then, we measure the voltage value at each load bus. If any of the bus voltage values are above 1.03 [p.u.] (for example), we have to perform voltage control. If all the load bus voltage values are below 1.03 [p.u.], we can install PV up to 30 [p.u.], which is the target value.

Fig. 6 shows a flowchart of static capacitor control. In Fig. 6, if any of the load bus voltage values are above 1.03 [p.u.], we calculate the VMPI sensitivity and make a bus number ranking in descending order of VMPI sensitivity. Finally, we reduce, by 0.05 [p.u.], the static capacitor installed at the bus with the highest ranking.

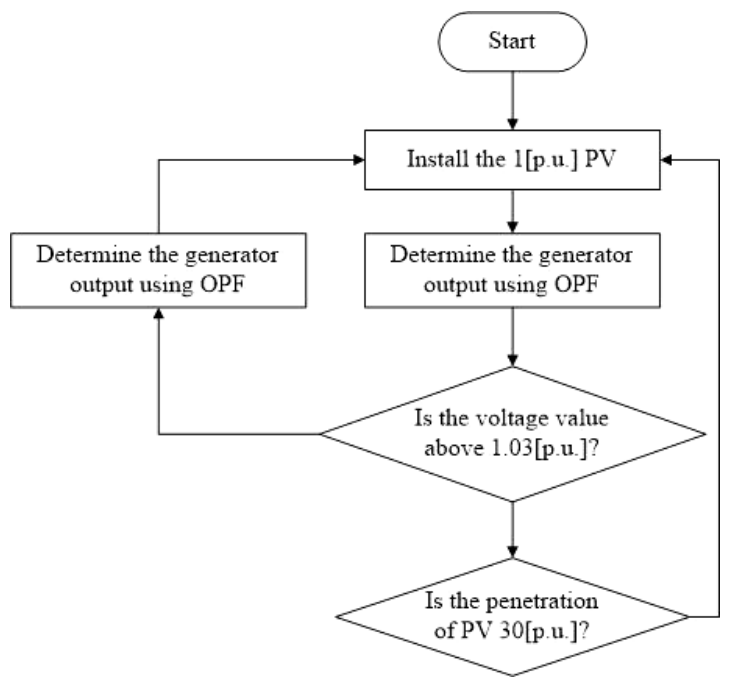

Fig. 5. Flowchart of PV penetration.

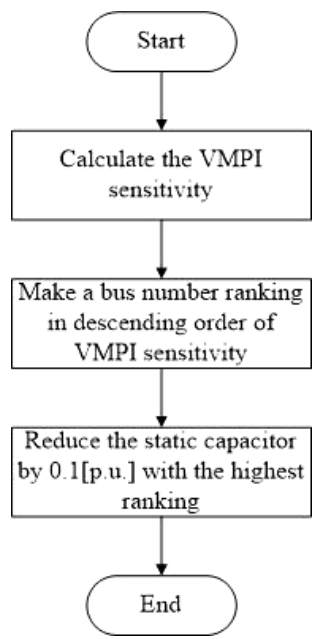

Fig. 6. Flowchart of the static capacitors control.

\section{Simulation}

In this section, a simulation is run to confirm the validity of the proposed method. In this simulation, the IEEJ WEST 10-machine O/V system model is used.

Assumptions in simulations are as follows.

1) PV is installed at all load buses, and the power output is proportional to each load demand.

2) The total generator active power outputs are equal to the total PV power outputs.

3) All generators are controlled and the reductions are proportional to generator's original active power outputs.

4) Control devices are only the static capacitors installed at the load buses. 
Fig. 7 shows the relationship between VMPI and the penetration of PV when the proposed method are carried out and Fig. 8 shows the voltage value at each load bus after voltage control has been carried out when the installed PV are at 30 [p.u.].

If we do not control the voltage, PV can be installed only to a maximum of 13 [p.u.]. But, by using the proposed method, we can install PV up to 30 [p.u.], which is our target. And Fig. 7 and Fig. 8 show the voltage value at each load bus after voltage control has been carried out. It is confirmed that the load bus voltages are within the proper voltage range.

Also, Table 1 shows the amount of each generator output control until 30 [p.u.] of PV are installed every 5 [p.u.]. Table 2 shows the static capacitors installed on each bus, which are used for voltage control until 30 [p.u.] of PV are installed. Method1 is that all generators are controlled and reductions are proportional to each generator's original active power output. Method2 is that all generators are controlled by proposed method. It was confirmed that amount of the static capacitor control is less than the method 1 when we determine the generator output using the proposed method.

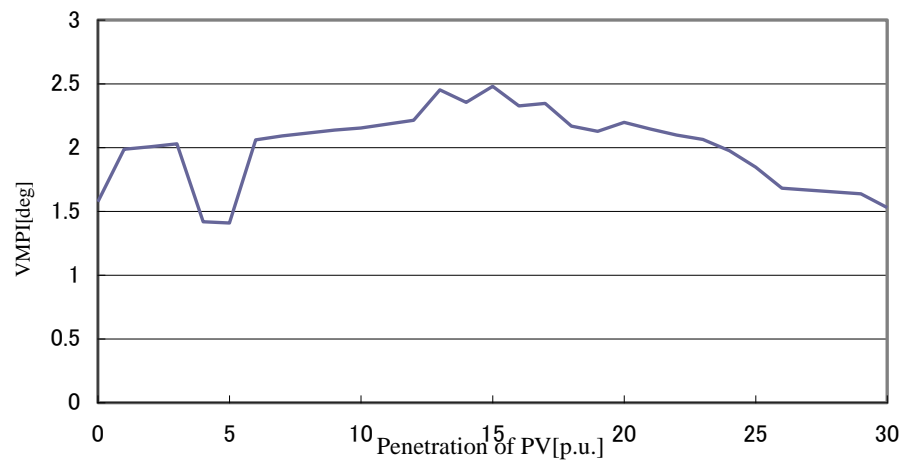

Fig. 7. Variation of VMPI for penetration of PV.

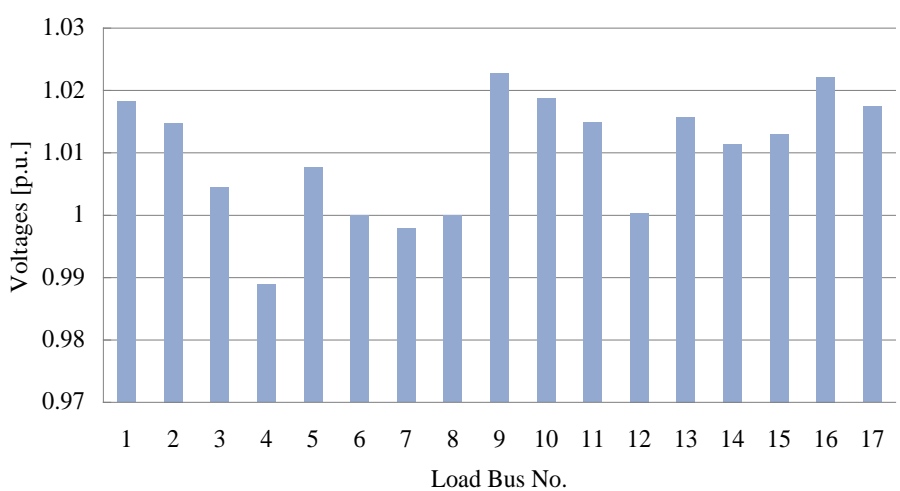

Fig. 8. Voltage value after the control.

Table 1. Amount of generator control

\begin{tabular}{ccccccc}
\hline \hline Generator & 5[p.u.] & 10[p.u.] & 15[p.u.] & 20[p.u.] & 25[p.u.] & 30[p.u.] \\
\hline G1 & -3.51 & -3.51 & -3.51 & -3.51 & -3.51 & -3.51 \\
G2 & -2.24 & -2.24 & -2.24 & -2.24 & -2.24 & -2.21 \\
G3 & 1.24 & -1.24 & -2.34 & -2.34 & -2.34 & -2.34 \\
G4 & 2.51 & 0.27 & -1.67 & -0.78 & -0.05 & -0.35 \\
G5 & -2.20 & -2.38 & 2.11 & 3.45 & 0.53 & -2.16 \\
G6 & 3.96 & 3.04 & 1.96 & 1.26 & -3.24 & -1.04 \\
G7 & -1.62 & 1.70 & 1.25 & -1.06 & -0.17 & -2.26 \\
G8 & -1.40 & -1.40 & -1.40 & -1.40 & -1.40 & -1.40 \\
G9 & 3.67 & -1.14 & -1.85 & -3.24 & -3.24 & -3.24 \\
G10 & -5.20 & -5.73 & -7.02 & -7.37 & -9.12 & -10.36 \\
\hline \hline
\end{tabular}


Table 2. Amount of static capacitor control

\begin{tabular}{cccc}
\hline \hline Bus No. & Initial value [p.u.] & Amount of control [p.u.] (Method 1) & Amount of control [p.u.] (Method 2) \\
\hline 3 & 0.35 & -0.10 & -0.05 \\
4 & 0.35 & -0.10 & 0.00 \\
5 & 0.35 & -0.10 & 0.00 \\
6 & 0.4 & -0.15 & 0.00 \\
7 & 0.65 & -0.25 & 0.00 \\
8 & 0.4 & -0.15 & 0.00 \\
9 & 3.5 & -0.05 & 0.00 \\
16 & 0.3 & -0.10 & 0.00 \\
\hline \hline
\end{tabular}

\section{Conclusion}

Recently, clean energy such as PV has attracted attention because of the threat posed by serious environmental and energy problems. Thus, in this paper, we have focused our attention on wide-scale penetration of $\mathrm{PV}$, with an emphasis on upper voltage limit considerations, and have shown the relationship between VMPI and PV penetration. Moreover, we have proposed a smart control method by using the optimal power flow in terms of minimizing bus voltage deviations from the prescribed values for a large penetration of PV. Finally, to verify the effectiveness of the proposed method, we have run simulations using the IEEJ WEST 10 machines O/V system model. As a result, it is confirmed that load bus voltages are within the proper voltage range in a small amount of static capacitor control by using the proposed method.

\section{References}

[1] Kataoka Y, Watanabe M, Iwamoto S. A new voltage stability index considering voltage limits. In: Proc. of IEEE PES PSCE '06, 2006: 1878-1883.

[2] Tamura Y, Sakemoto K, Tayama Y. Voltage instability proximity index (VIPI) based on multiple load flow solutions in illconditioned power systems. In: Proc. of the 27th IEEE Conference on Dicision and Control, 1988:2114-2119.

[3] Kataoka Y, Watanabe M, Iwamoto S. Voltage stability preventive control using VMPI sensitivities. Presented at: IEEJ Proceedings of the 18th Annual Conference of Power \& Energy Society, 2006 (in Japanese).

[4] Sakaeda S, Watanabe M, Iwamoto S. Fast voltage stability preventive control using ellipse-approximate P-V curves. Presented at: 2008 IEEE PES General Meeting, 2008.

[5] Tanaka H, Tokumitsu K, Iwamoto S, Kobayashi R, Hirano D, Takeuchi A. Development of the emergency voltage control scheme using VMPI. Presented at: 2009 IEEE PES General Meeting, 2009.

[6] Standing Special Committee of Power System Model Standardization. Standard Model of Power Systems. Technical Report of IEE of Japan, No. 754, 1999. 